\title{
ANÁLISE PERFILOMÉTRICA DO PADRÃO DE DESGASTE DENTÁRIO DE INDIVÍDUOS BRUXÔMANOS E SUA INTER-RELAÇÃO COM A EROSÃO DENTÁRIA: UM ESTUDO LONGITUDINAL
}

\section{Letícia Ladeira Bonato}

Doutorado em andamento pela Universidade Federal Fluminense - UFF, Brasil.

E-mail: leticialbonato@hotmail.com

\section{Flávia de Souza Bastos}

Docente Adjunta II do Departamento de Mecânica Aplicada e Computacional da Universidade Federal de Juiz de Fora - UFJF, Brasil.

\section{Agnes Batista Meireles}

Doutora em Bioengenharia pela Universidade Federal de Minas Gerais - UFMG, Brasil.

\section{Renato Françoso de Ávila}

Pós-doutorado sênior realizado na Faculdade de Engenharia Mecânica da Universidade Federal de Uberlândia - UFU, Brasil.

\section{Josemar Parreira Guimarães}

Doutor em Ortodontia pela Faculdade de Odontologia da Universidade Federal do Rio de Janeiro - UFRJ, Brasil; Coordenador do Serviço de Diagnóstico e Orientação a Pacientes com Desordens Temporomandibulares.
RESUMO: O desgaste dentário é considerado um processo fisiológico. Contudo, quando o mesmo apresenta-se acentuado, frequentemente o bruxismo é apontado como o único fator etiológico envolvido, desconsiderando-se outros possíveis padrões de desgaste, como a erosão e a abrasão. O propósito deste estudo foi avaliar através da análise da rugosidade superficial de dentes como é o padrão de desgaste dentário de mulheres diagnosticadas com erosão ácida e/ou atrição. Trata-se de um estudo descritivo observacional longitudinal. A amostra foi constituída por 20 mulheres entre 20 e 50 anos, divididas em grupo A $(n=9)$, caracterizado por integrantes que realizavam o bruxismo, e grupo $B(n=11)$, formado por mulheres que não realizavam tal função. Uma subdivisão foi ainda realizada, separando aquelas que possuíam fatores erosivos em sua dieta. Para obtenção das réplicas dentárias das pacientes, foi realizada moldagem da arcada superior com Silicona de Adição após minucioso exame clínico. Para análise da rugosidade superficial das amostras, foi utilizado o Rugosímetro Mitutoyo Surftest SJ-301. Todas as participantes do grupo A e B com fatores erosivos presentes apresentaram aumentos no parâmetro $\mathrm{Ra}$, da ordem de $25,16 \%, 200 \%$ e $74,9 \%$, respectivamente. O grupo B sem erosão foi o único em que a diferença observada não pode ser considerada significativa. Em relação à curva de material AbbotFirestone, a diferença apresentou-se acentuada apenas nos grupos onde havia a presença de fatores erosivos. Os resultados mostraram que o padrão de desgaste dentário quando presentes fatores erosivos é acentuado quando comparado com o desgaste decorrente apenas da atrição.

PALAVRAS-CHAVE: Bruxismo; Desgaste Dentário; Erosão Dentária.

\section{PROFILE ANALYSIS OF STANDARD TEETH WEAR IN PEOPLE SUFFERING FROM BRUXISM AND ITS RELATIONSHIP WITH DENTAL EROSION: A LONGITUDINAL ANALYSIS}

\begin{abstract}
Although teeth wear is a physiological process, bruxism or teeth grinding is indicated as the sole etiological factor involved, without taking into consideration other wear processes, such as erosion and abrasion. Current descriptive and longitudinal analysis evaluates the surface roughness in teeth as dental wear standard in females diagnosed with acid erosion or attrition. Sample comprised 20 females, aged between 20 and 50 years old, divided into Group A $(n=9)$ characterized by teeth grinding, and Group B $(n=11)$ without bruxism. A subdivision was also included by dividing those with erosion factors in the diet. After a thorough exam, molds of the upper teeth with Addition Silicon Impression were undertaken to obtain the patients' dental replicas. Mitutoyo Surface Roughness Analyzer SJ-
\end{abstract}


301 was employed to investigate the samples' surface roughness. All participants of Groups A and B with erosion factors had an increase in $\mathrm{Ra}$ at $25.16 \%, 200 \%$ and $74.9 \%$, respectively. Erosion-less Group B was the only one with a non-significant difference. Difference in the Abbot-Firestone curve was sharp only in groups with erosion factors. Results revealed that the standard of dental wear with erosion factors was high when compared to attrition-caused wear.

KEY WORDS: Bruxism; Dental Wear; Teeth Erosion.

\section{INTRODUÇÃO}

Mesmo diante da crescente prevenção de doenças sistêmicas e orais (TAVARES et al., 2007), uma das alterações mais comumente encontradas em diversas populações é o desgaste dentário (HOLBROOK; ÁRNADÓTTIR; KAY, 2003; TAVARES et al., 2007). Este fenômeno ocorre fisiologicamente durante toda a vida, de forma lenta e progressiva, representando a adaptação do sistema mastigatório às funções cotidianas; entretanto este processo pode alterar-se para um padrão patológico (RANJITKAR, 2009). Grande perda tecidual (de esmalte e dentina), hipersensibilidade dentinária, exposição pulpar (OGLE; DAVIS, 1998; GANSS, 2006), perda da dimensão vertical de oclusão, potencial mastigatório deficiente, alteração nas relações de contato entre dentes e músculos mastigatórios fadigados podem ser consequências do desgaste dentário excessivo (OGLE; DAVIS, 1998; GHAZAL et al., 2008).

O fenômeno do desgaste ocorre sempre que uma superfície movimenta-se relativamente quando em contato com uma outra, ou quando exposta a substâncias quimicamente ativas (HEINTZE; ZAPPINI; ROUSSON, 2005), sendo consequência da interação de diversos fatores (HOLBROOK; ÁRNADÓTTIR; KAY, 2003; GANSS, 2006). Em Odontologia, os termos utilizados para os principais fenômenos do desgaste dentário são: atrição (SMITH; BARTLETT; ROBB, 1997; HOLBROOK; ÁRNADÓTTIR; KAY, 2003; BARDLEY; TAYLOR; MILOSEVIC, 2004; BARTLETT, 2005; BARTLETT; SHAH, 2006; GANSS, 2006; RANJITKAR, 2009; DALY, 2010; BHUSHAN et al., 2011; JOHANSSON et al., 2012); perda tecidual devido ao íntimo contato dente-dente (KAIDONIS et al.,
2003; GANSS, 2006; JOHANSSON et al., 2012; SMITH; BARTLETT; ROBB, 1997); abrasão (SMITH; BARTLETT; ROBB, 1997; HOLBROOK; ÁRNADÓTTIR; KAY, 2003; BARDLEY; TAYLOR; MILOSEVIC, 2004; BARTLETT, 2005; BARTLETT; SHAH, 2006; GANSS, 2006; RANJITKAR, 2009; DALY et al., 2010; BHUSHAN et al., 2011; JOHANSSON et al., 2012); resultante do atrito de material exógeno forçado sobre as superfícies dos dentes, como, por exemplo, a mastigação de alimentos (SMITH; BARTLETT; ROBB, 1997; KAIDONIS et al., 2003; GANSS, 2006; JOHANSSON et al., 2012); e erosão (SMITH; BARTLETT; ROBB, 1997; HOLBROOK; ÁRNADÓTTIR; KAY, 2003; BARDLEY; TAYLOR; MILOSEVIC, 2004; BARTLETT, 2005; BARTLETT; SHAH, 2006; GANSS, 2006; RANJITKAR, 2009; DALY et al., 2010; BHUSHAN et al., 2011; JOHANSSON et al., 2012); perda de tecidos duros do dente por ação química que não envolvem bactérias (SMITH; BARTLETT; ROBB, 1997; KAIDONIS et al., 2003; BARTLETT; SHAH, 2006; JOHANSSON et al., 2012). A distinção entre o desgaste causado por estes fenômenos não é uma tarefa fácil para o profissional (SMITH; BARTLETT; ROBB, 1997; BARDLEY; TAYLOR; MILOSEVIC, 2004; BARTLETT; SHAH, 2006; GANSS, 2006) ocorrendo muitas vezes de forma combinada com diferentes intensidades e durações, resultando distintos padrões e graus de perda tecidual (KAIDONIS et al., 2003).

Considerando-se o aumento da prevalência geral do bruxismo, frequentemente durante a atividade clínica, quando verificadas lesões de desgaste, estas são por inferência atribuídas apenas a este hábito parafuncional (KHAN; YOUNG; DALEY, 1998). O bruxismo inclui o rangimento (excêntrico) e/ou apertamento dentário (cêntrico), podendo apresentar-se sem sinais, sintomas ou efeitos nocivos ao sistema mastigatório, mas podendo tornar-se problemático ao contribuir para um aumento no desgaste dos dentes, surgimento de dor (KHAN; YOUNG; DALEY, 1998; LURIE et al., 2007; BARBOSA et al., 2008) e desenvolvimento de desordens temporomandibulares (DTM) (KAIDONIS et al., 2003; BARBOSA et al., 2008).

Parte das lesões apresentadas por pacientes bruxômanos pode, de fato, ser devido à atrição, no entanto uma alta incidência de lesões erosivas também pode ser encontrada nas superfícies oclusais destes pacientes (KHAN; YOUNG; DALEY, 1998). Atualmente, 
dentre os fenômenos capazes de promover o desgaste dentário, a erosão é considerada a manifestação clínica mais importante (WHITEHEAD et al., 1999; HOLBROOK; ÁRNADÓTTIR; KAY, 2003; BARTLETT, 2005; DALY et al., 2010). Provavelmente, a extensão das lesões de desgaste, principalmente em grupos etários jovens, é muito maior quando a erosão é a responsável, quando comparados com outras causas (SMITH; BARTLETT; ROBB, 1997). As constantes mudanças nos estilos de vida e alimentares da população e o aumento crescente no consumo de alimentos e bebidas ácidas contribuíram para tal fato (WHITEHEAD et al., 1999; BARTLETT, 2005), possuindo altas prevalências de lesões erosivas em todas as partes do mundo, atingindo inclusive crianças e adolescentes (JOHANSSON et al., 2012). Com a desmineralização ácida do dente, são formadas lesões em forma de concha nas regiões incisais e de pontas de cúspides, sendo três os principais promotores da erosão dentária (HOLBROOK; ÁRNADÓTTIR; KAY, 2003; BARTLETT, 2005; DALY et al., 2010): a dieta, alterações gastro-intestinais (HOLBROOK; ÁRNADÓTTIR; KAY, 2003; BARTLETT, 2005; DALY et al., 2010; JOHANSSON et al., 2012) e o contato com ácidos industrializados (DALY et al., 2010). A taxa de fluxo salivar e a capacidade tampão da saliva também podem influenciar o processo erosivo (SMITH; BARTLETT; ROBB, 1997; YOUNG; KHAN, 2002). Vale ressaltar que a erosão pode predispor o indivíduo a realizar quadros de atrição grave, onde os dois mecanismos passam a atuar simultaneamente causando o desgaste do dente (KHAN; YOUNG; DALEY, 1998).

A perfilometria tem sido utilizada na tentativa de que mudanças na superfície causadas, por exemplo, pela parafunção possam ser detectadas mais precocemente (LAS CASAS et al., 2006). É um dos principais sistemas capazes de mapearem a superfície dentária, permitindo a mensuração de parâmetros geométricos da superfície de amostras submetidas ao fenômeno do desgaste (RODRIGUEZ; AUSTIN; BARTLETT, 2012). Aproximadamente 100 variantes para avaliar a rugosidade superficial podem estar envolvidas, sendo que na Odontologia um dos parâmetros mais utilizados para analisar a topografia é a Rugosidade média (Ra) (MEIRELES, 2009), definida como a média aritmética das amplitudes encontradas em um perfil (WHITEHEAD et al., 1999; RIOS et al., 2002). Contudo, apesar de o parâmetro "Ra" informar a amplitude verdadeira de um perfil, não fornece qualquer informação sobre as características texturais do mesmo (WHITEHEAD et al., 1999; FIELD; WATERHOUSE; GERMAN, 2010), a probabilidade de desgaste futuro ou sua resistência ao desgaste (FIELD; WATERHOUSE; GERMAN, 2010). Por isso, outra forma de descrever o perfil é traçando suas características superficiais em uma curva onde podem ser comparados quantitativa e qualitativamente com outros perfis superficiais, sendo utilizados para isso os parâmetros da família "Rk" (PETROPOULOS, 2007; BASTOS, 2008). Ao se analisar o perfil de uma superfície rugosa, pode-se imaginar a interseção desta com uma linha de referência que desce desde o pico mais alto até o vale mais profundo (Figura 1). Para cada profundidade, "c", a taxa de material, $\operatorname{Rmr}(\mathrm{c})$, é obtida através da soma dos comprimentos de material, MI(c) (região de interseção entre a superfície rugosa e a linha de referência). A curva de material Abbot-Firestone é o gráfico das taxas de material $\operatorname{Rmr}(c)$ em função da profundidade, c. Esta curva, que relaciona a transição entre ar e material, permite a comparação quantitativa e qualitativa entre diferentes perfis. Ela pode ser, então, subdivida em três regiões delimitadas pelos parâmetros da família Rk (Figura 1), definidos como:

- Rk: amplitude da rugosidade na zona núcleo;

- Rpk: amplitude acima da rugosidade na zona núcleo;

- Rvk: amplitude abaixo da rugosidade na zona núcleo;

- MR1 e MR2: representam as taxas de material encontradas nos extremos da zona núcleo.

Com isso, o presente artigo propôs-se a avaliar, através da análise do perfil de rugosidade superficial de dentes, como é o padrão de desgaste dentário de mulheres diagnosticadas com erosão ácida e/ou atrição.

\section{METODOLOGIA}




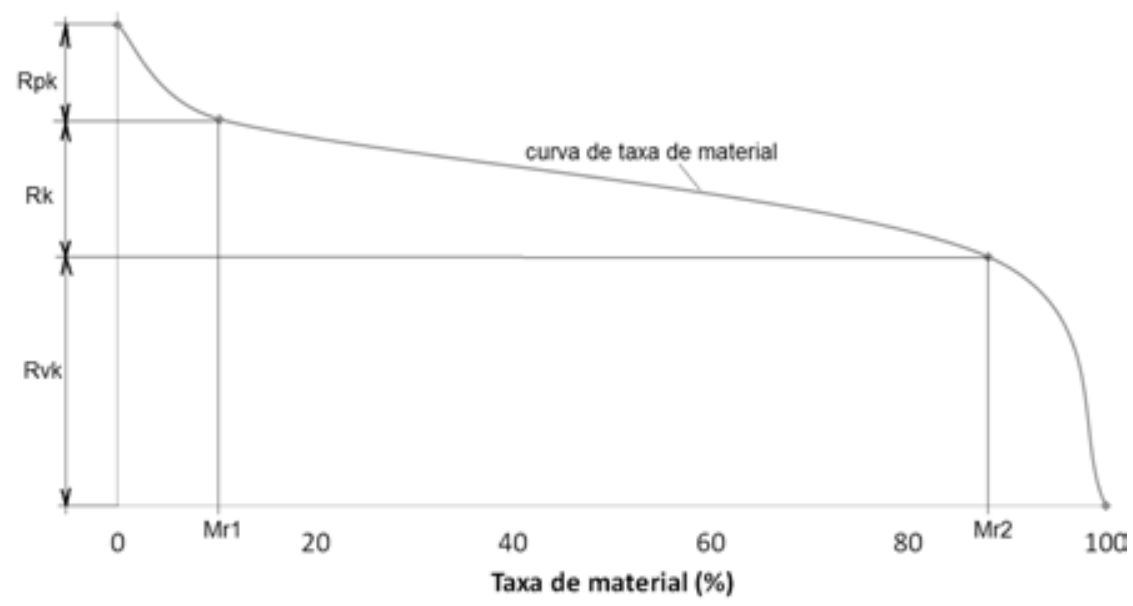

Figura 1. Parâmetros da Família "Rk"

Trata-se de um estudo descritivo observacional transversal, aprovado pelo Comitê de Ética em Pesquisa da Universidade Federal de Juiz de Fora, sob o parecer número 2450.190.2011. O consentimento de participação dos pacientes foi registrado em termo assinado anteriormente à execução da pesquisa.

A composição da amostra contou com a participação de mulheres pertencentes à faixa etária compreendida entre 20 e 50 anos (AHLBERG et al., 2002). Os critérios de inclusão da pesquisa basearamse na ausência de doenças sistêmicas (como diabetes, artrite, fibromialgia e outras), não fazer uso de fármacos tais quais inibidores seletivos da receptação de serotonina, catecolaminas, anfetaminas, dopaminas, cafeína, antidepressivos tricíclicos, fenilalanina, agonista da serotonina, antipsicóticos e ansiolíticos (como os Benzodiazepínicos), além de relaxantes musculares, não estar sob terapia ortodôntica e/ou para DTM e possuir todos os dentes (com exceção dos terceiros molares). As mulheres que não apresentaram essas características não fizeram parte do estudo.

Para a realização do estudo, uma ficha de avaliação contendo os dados da participante foi utilizada, sendo que após o preenchimento da mesma, cinco etapas foram seguidas:

\section{$1^{\text {a }}$ etapa - Diagnóstico do bruxismo}

Conforme recomendações da Classificação Internacional de Desordens do Sono (ICSD) (GHAZAL et al., 2008), quatro questões foram adaptadas e respondidas pelo voluntário: A) Você realiza apertamento e/ou rangimento dentário durante o dia, principalmente quando está realizando atividades estressantes ou que exigem muita concentração?; B) Apresenta uma ou mais das seguintes situações: desgaste anormal dos dentes, sons associados com o bruxismo ou desconforto muscular?; C) Apresenta alguma desordem médica ou mental? (de origem psiquiátrica ou neurológica, por exemplo); 4) Apresenta algum tipo de distúrbio do sono? (por exemplo, insônia, apneia, ronco ou despertares). Para ser considerado um bruxômano do sono, pelo menos as questões A e B deviam ser respondidas positivamente.

\section{$2^{\mathrm{a}}$ etapa - Análise de fatores de risco para doenças do refluxo gastro-esofágico}

Durante esta etapa, todas as participantes deveriam responder a um questionário minucioso (CORRÊA, 2007), contendo perguntas relativas à sua saúde geral e sintomas relacionados às doenças do refluxo gastresofágico, contendo as questões: A) Você se alimenta antes de dormir?; B) Ingere sucos, frutas cítricas ou xaropes melados? Qual o tipo e frequência?; C) Toma refrigerantes, bebidas esportivas ou drinques? Qual e com que frequência?; D) Tem azia, vômito, gosto azedo na boca ou alguma desordem alimentar?; E) Faz uso de algum tipo de droga atualmente? Qual e há quanto tempo?; F) Tem o hábito de tomar algum remédio? Qual?; G) Há quanto tempo foi ao dentista? O profissional notou algum desgaste em seus dentes?; H) Você sabe que possui tais lesões (desgastes)?; I) Quais são seus hábitos 
de higiene oral (como escova os dentes, tipo de escova, quantas vezes ao dia?); J) Usa solução fluoretada (ex: pasta de dente, líquidos para bochechos, dentre outros)? Qual?; L) Tem regurgitação? Com que frequência e há quanto tempo?; M) Tem vômitos? Qual a frequência e há quanto tempo?; N) Se possui algum sinal mencionado, já foi ao médico ou toma medicamentos por conta própria?

\section{$3^{\mathrm{a}}$ etapa - Mensuração do $\mathrm{pH}$ salivar}

Após lavar a boca com água corrente para eliminar descamação epitelial e restos bacterianos, as voluntárias foram orientadas a não deglutir a saliva durante um período de 5 minutos. Esta saliva deveria escorrer pela boca (evitando-se escarrar para não trazer material da rinofaringe) para um copo descartável. $\mathrm{O}$ pH da saliva foi avaliado a partir de uma fita colorimétrica da marca Macherey-Nagel ${ }^{\circledR}$ (MachereyNagel - Brasil), que deveria ficar submerso na solução durante 10 minutos.

As participantes foram orientadas a não realizarem nenhuma atividade que pudesse estimular o fluxo salivar cerca de $1 \mathrm{~h} 30$ a $2 \mathrm{~h}$ antes da coleta (como beber ou comer, mastigar, fumar, escovar os dentes e realizar bochechos); a coleta da saliva foi realizada com a participante sentada relaxadamente na cadeira odontológica da instituição de ensino, respeitando o mesmo horário de coleta entre as mesmas.

$4^{\mathrm{a}}$ etapa - Obtenção de réplicas parciais da arcada superior e análise do perfil de rugosidade
Na quarta etapa, a moldagem foi realizada, devendo envolver a região de canino superior do lado direito a incisivos central e lateral também do lado direito (utilizando para isso uma moldeira parcial perfurada), objetivando-se obter a cópia da topografia superficial dos dentes. Para obtenção do modelo, a moldagem foi realizada utilizando a Silicona de Adição (President ${ }^{\circledR}$ ), e as réplicas positivas, utilizando resina Flow (3M). Aguardou-se por duas horas para a fase de cura, destacando a réplica da moldeira. As participantes da pesquisa foram moldadas por um período de 45 dias, de 15 em 15 dias, obtendo três réplicas de cada paciente em cada visita, para que fossem evitados erros de moldagem. Após a obtenção da réplica, foi feito o corte da mesma, utilizando disco diamantado, centralizando a faceta desgastada de modo a obter uma amostra o mais plana possível. Em seguida, o embutimento da amostra em resina acrílica foi realizado, com o objetivo de facilitar o posicionamento da amostra em relação ao equipamento, evitando o seu movimento durante a medição.

Para a análise da rugosidade superficial das amostras, foi utilizado o Rugosímetro Mitutoyo Surftest SJ-301 (Japão) (Figura 2), onde um padrão relativo ao posicionamento da amostra e do braço do rugosímetro foi seguido, obtendo um contato apropriado da ponta do rugosímetro com a superfície a ser analisada (Figura 3). A medida da rugosidade foi realizada utilizando um filtro de rugosidade $0,05 \mathrm{~mm}$, realizando cinco varreduras por amostra. Foram utilizados os parâmetros Rugosidade média (Ra) (MEIRELES, 2009), e parâmetros da família Rk, sendo eles: Rk, Rpk, Rvk, Mr1 e Mr2.

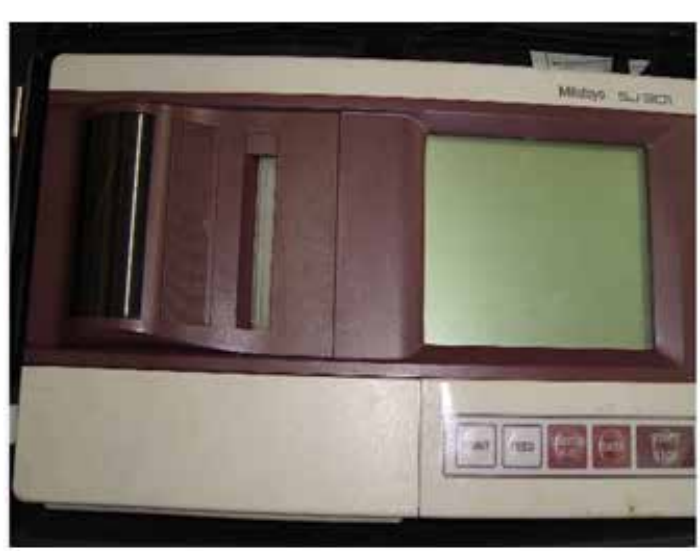

$2 A$

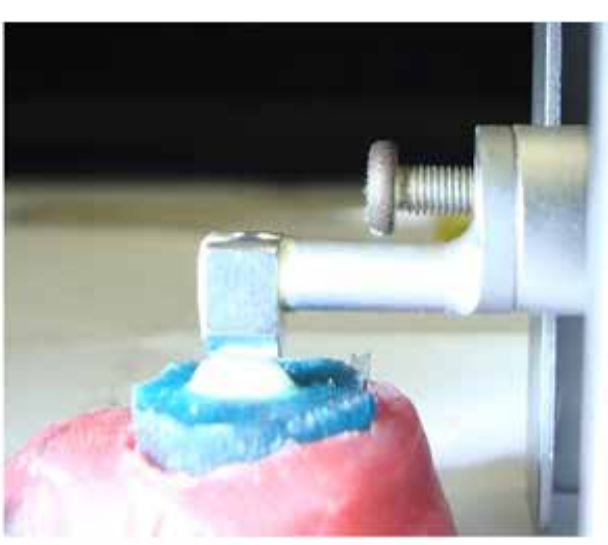

2B

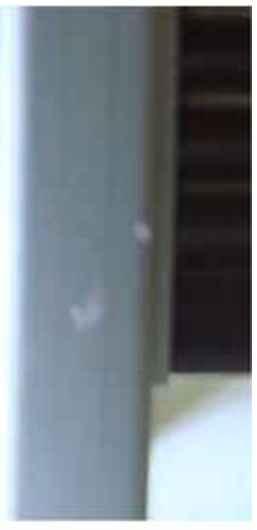

B) Braço de

Figura 2. Aparelho Rugosímetro Mitutoyo Surftest SJ-301 utilizado para realizar as mensurações: A) Aparelho visão superficial; B) Braço de leitura com amostra posicionada. 


\section{RESULTADOS}

Inicialmente, 25 mulheres foram incluídas no estudo. Contudo, cinco participantes não puderam continuar por não preencherem os critérios de inclusão. Com isso, o estudo contou com a participação de 20 integrantes. A média de idade encontrada foi de 32,6 anos $( \pm 7,3)$.

A amostra foi então dividida em dois grupos, sendo estes o grupo A e o grupo B. O grupo A foi composto por nove integrantes diagnosticadas como bruxômanas, sendo que em quatro delas detectaram-se fatores sugestivos de erosão. Já o grupo B, constituiuse de onze participantes diagnosticadas como não bruxômanas, sendo que também em quatro delas foram detectados possíveis fatores erosivos.

Foram avaliados os parâmetros Ra inicial $\left(\mathrm{Ra}_{\mathrm{i}}\right)$ e final $\left(\mathrm{Ra}_{\mathrm{f}}\right)$ de todas as integrantes e comparados com a variação do $\mathrm{pH}$ (também inicial e final) das mesmas. Na Tabela 1 estão apresentados os resultados das cinco participantes do grupo A que não apresentavam fatores sugestivos de erosão em sua dieta, as quais apresentaram a diferença de valores de $\mathrm{Ra}$ (Raf - Rai) em porcentagem respectivamente de $30,4 \% ; 11,1 \% ; 53,3 \% ; 16,7 \%$ e $14,3 \%$. A diferença entre o $\mathrm{pH}$ final e o inicial foram neste grupo de respectivamente $0 ; 1 ;-1 ;-1$ e 0 (Tabela 1 ).

Tabela 1. Valores iniciais e finais para os parâmetros Ra e pH das integrantes do grupo $\mathrm{A}$

\begin{tabular}{ccccccc}
\hline $\begin{array}{c}\text { Grupo A } \\
\text { (Sem erosão) }\end{array}$ & $\mathbf{R a}_{\mathbf{i}}$ & $\mathbf{R a}_{\mathbf{f}}$ & $\begin{array}{c}\mathbf{R a}_{\mathrm{f}}-\mathbf{R a}_{\mathbf{i}} \\
(\%)\end{array}$ & $\mathbf{P h}_{\mathrm{i}}$ & $\mathbf{P h}_{\mathbf{f}}$ & $\begin{array}{c}\mathbf{P h}_{\mathrm{f}} \\
\mathbf{P h}_{\mathbf{i}}\end{array}$ \\
\hline Participante 1 & 0,23 & 0,3 & 30,4 & 7 & 7 & 0 \\
Participante 2 & 0,27 & 0,3 & 11,1 & 7 & 8 & 1 \\
Participante 3 & 0,15 & 0,23 & 53,3 & 8 & 7 & -1 \\
Participante 4 & 0,54 & 0,63 & 16,7 & 7 & 6 & -1 \\
Participante 5 & 0,35 & 0,4 & 14,3 & 8 & 8 & 0 \\
\hline
\end{tabular}

Avaliou-se também neste grupo os casos em que os fatores erosivos foram evidenciados. As participantes do grupo A que apresentaram fatores sugestivos de erosão obtiveram a diferença de valores de $\mathrm{Ra}$ (Ra final menos $\mathrm{Ra}$ inicial) de respectivamente $126 \%$; $450 \%$; 68,75\% e $154 \%$, e diferença de $\mathrm{pH}$ (final menos o inicial) de $-1 ;-2 ;-1$ e 1 (Tabela 2).
Tabela 2. Valores iniciais e finais para os parâmetros $\mathrm{Ra}$ e $\mathrm{pH}$ da participante do grupo A que possuía possíveis fatores erosivos e sua dieta

\begin{tabular}{ccccccc}
\hline $\begin{array}{c}\text { Grupo A } \\
\text { (com erosão) }\end{array}$ & Rai & Raf & $\begin{array}{c}\text { Raf - Rai } \\
\text { (\%) }\end{array}$ & Phi & Phf & $\begin{array}{c}\text { Phf } \\
\text {-Phi }\end{array}$ \\
\hline Participante 6 & 0,23 & 0,52 & 126 & 8 & 7 & -1 \\
Participante 7 & 0,1 & 0,55 & 450 & 8 & 6 & -2 \\
Participante 8 & 0,32 & 0,54 & 68,75 & 7 & 6 & -1 \\
Participante 9 & 0,24 & 0,61 & 154 & 7 & 8 & 1 \\
\hline
\end{tabular}

Já no grupo B (considerado controle), dentre as participantes, sete não possuíram fatores sugestivos de erosão em sua dieta, sendo encontrada uma diferença de valores de $\mathrm{Ra}$ (Raf - Rai) respectivamente de -5\%; 4,6\%; $4,6 \% ; 3 \% ; 10 \% ; 9,7 \%$ e $17,9 \%$. A diferença entre o $\mathrm{pH}$ final e o inicial neste grupo foram respectivamente de $1 ; 0 ; 0$; $0 ; 1 ; 0$ e 0 (Tabela 3).

Tabela 3. Valores iniciais e finais para os parâmetros $\mathrm{Ra}$ e $\mathrm{pH}$ das participantes do grupo B

\begin{tabular}{lcccccc}
\hline $\begin{array}{c}\text { Grupo B } \\
\text { (sem atrição e } \\
\text { sem erosão) }\end{array}$ & $\mathbf{R a}_{\mathbf{i}}$ & $\mathbf{R a}_{\mathbf{f}}$ & $\begin{array}{c}\mathbf{R a}_{\mathbf{f}}- \\
\left.\mathbf{R a}_{\mathbf{i}} \mathbf{~}^{-}\right)\end{array}$ & $\mathbf{P h}_{\mathbf{i}}$ & $\mathbf{P h}_{\mathbf{f}}$ & $\begin{array}{c}\mathbf{P h}_{\mathbf{f}} \\
\mathbf{- P h}_{\mathbf{i}}\end{array}$ \\
\hline Participante 1 & 0,2 & 0,19 & -5 & 6 & 7 & 1 \\
Participante 2 & 0,22 & 0,23 & 4,6 & 7 & 7 & 0 \\
Participante 3 & 0,22 & 0,23 & 4,6 & 7 & 7 & 0 \\
Participante 4 & 0,33 & 0,34 & 3 & 7 & 7 & 0 \\
Participante 5 & 0,2 & 0,22 & 10 & 6 & 7 & 1 \\
Participante 6 & 0,31 & 0,34 & 9,7 & 8 & 8 & 0 \\
Participante 7 & 0,28 & 0,33 & 17,9 & 7 & 7 & 0 \\
\hline
\end{tabular}

Ainda neste grupo, dentre as participantes que apresentaram fatores erosivos possivelmente presentes, a diferença de valores de $\mathrm{Ra}$ (Ra final menos Ra inicial) encontrada foi de 56,3\%; 63,3\%; 88,5\% e 91,3\% e diferença de $\mathrm{pH}$ de $-1 ;-1 ;-1$ e -2 (Tabela 4 ).

Tabela 4. Valores iniciais e finais para os parâmetros Ra e $\mathrm{pH}$ da participante do grupo B que possuía possíveis fatores erosivos e sua dieta

\begin{tabular}{lcccccc}
\hline $\begin{array}{c}\text { Grupo B } \\
\text { (sem atrição e } \\
\text { com erosão) }\end{array}$ & $\mathbf{R a}_{\mathbf{i}}$ & $\mathbf{R a}_{\mathbf{f}}$ & $\begin{array}{c}\mathbf{R a}_{\mathbf{f}} \mathbf{}_{\mathbf{6}} \\
\mathbf{R a}_{\mathbf{i}} \% \text { ) }\end{array}$ & $\mathbf{P h}_{\mathbf{i}}$ & $\mathbf{P h}_{\mathbf{f}}$ & $\begin{array}{c}\mathbf{P h}_{\mathbf{f}} \\
\mathbf{P h}_{\mathbf{i}}\end{array}$ \\
\hline Participante 8 & 0,16 & 0,25 & 56,3 & 8 & 7 & -1 \\
Participante 9 & 0,3 & 0,49 & 63,3 & 8 & 7 & -1 \\
Participante 10 & 0,26 & 0,49 & 88,5 & 7 & 6 & -1 \\
Participante 11 & 0,23 & 0,44 & 91,3 & 8 & 6 & -2 \\
\hline
\end{tabular}


Foram ainda traçadas linhas de tendência obtidas através dos pontos da curva de material nos instantes inicial e final de cada subgrupo, sendo eles Grupo A, com erosão e sem erosão, e Grupo B também com e sem erosão. As linhas contínuas referem-se ao instante inicial, e as linhas tracejadas representam o instante final (Gráfico 1).

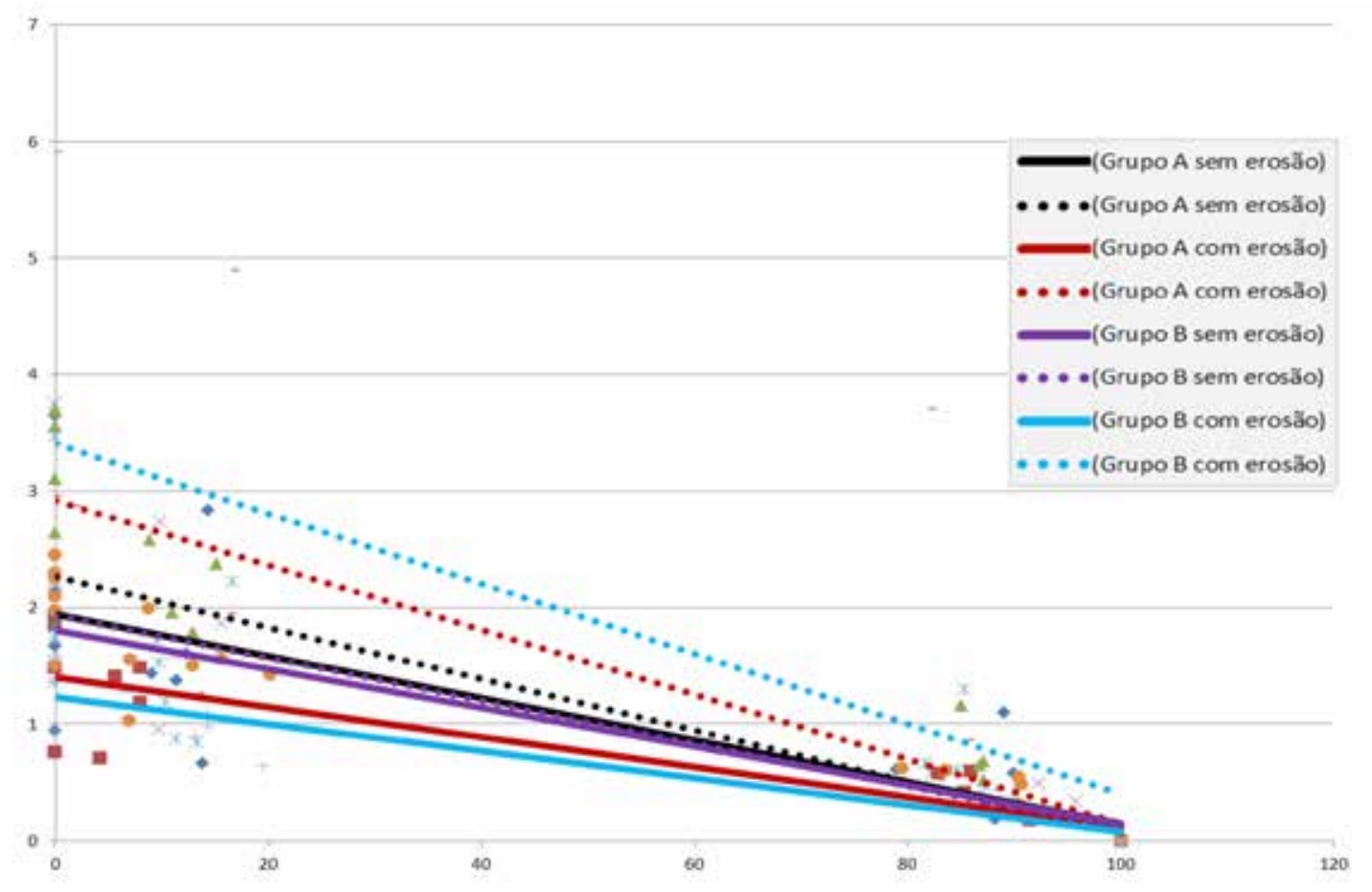

Gráfico 1. Linhas de tendência nos instantes inicial e final.

\section{DISCUSSÃO}

Apesar de o desgaste dentário ser o sinal mais frequentemente encontrado em pacientes bruxômanos (KHAN; YOUNG; DALEY, 1998; PERGAMALIAN et al., 2003; LURIE et al., 2007; BARBOSA et al., 2008), sua etiologia multifatorial, a avaliação subjetiva de sua presença, e um padrão complexo natural do bruxismo, fazem com que a relação entre este hábito parafuncional e o desgaste dentário ainda seja controversa na literatura (PERGAMALIAN et al., 2003). Durante muito tempo, fatores periféricos e morfológicos foram relacionados à etiologia deste hábito, sendo as discrepâncias oclusais e a anatomia das estruturas ósseas da região orofacial considerados como os principais fatores causadores do bruxismo. Atualmente, estes fatores desempenham um papel menos importante na etiologia do mesmo, sendo o foco voltado aos fatores centrais (LOBEZOO; NAEIJE, 2001; BARBOSA et al., 2008).
Alterações químicas cerebrais (LOBEZOO; NAEIJE, 2001), processos alérgicos, infecções de vias aéreas (GRECHI et al., 2008), traumas, doenças neuropsiquiátricas (podendo acarretar movimentos faciais anormais) (SALVI et al., 2009), e outras doenças são exemplos de alterações do sistema nervoso central que podem estar envolvidas na precipitação do bruxismo (LOBEZOO; NAEIJE, 2001). Com isso, para que fossem afastadas variáveis que pudessem influenciar o padrão de realização do hábito parafuncional em questão, as participantes deste estudo não deveriam apresentar qualquer tipo de doença sistêmica (LOBEZOO; NAEIJE, 2001) (como diabetes, artrite, fibromialgia e outras). Também foi restringido o uso das medicações tais quais inibidores seletivos da receptação de serotonina, catecolaminas, anfetaminas, dopaminas, cafeína, antidepressivos tricíclicos, fenilalanina, agonista da serotonina, antipsicóticos e ansiolíticos (como os Benzodiazepínicos), além de relaxantes musculares uma 
vez que algumas drogas, principalmente as que atuam no âmbito do Sistema Nervoso Central, são capazes tanto de exacerbar o bruxismo - quadro conhecido como distonia induzida por drogas, sendo este tipo de bruxismo uma desordem distinta do de origem idiopática (ORTEGA, 2009), quanto atenuar os quadros do mesmo (LOBEZOO; NAEIJE, 2001; ORTEGA, 2009). Como o desgaste dentário pode estar presente também durante a realização de tratamentos ortodônticos (GRECHI et al., 2008), contraindicou-se a realização conjunta com o período da pesquisa em questão, assim como terapia para DTM.

Através do método de perfilometria por contato, foram obtidas cerca de 20 variáveis distintas para cada amostra avaliada, no entanto algumas destas variáveis foram selecionadas e avaliadas criteriosamente para que o fenômeno tanto do desgaste dentário quanto da erosão pudessem ser analisados e interpretados de forma correta. Dentre os parâmetros escolhidos, está o parâmetro Ra, o qual informa sobre a dispersão das alturas das asperezas em relação ao plano médio da superfície. Em nosso estudo, esperávamos que tanto os indivíduos bruxômanos quanto aqueles que relatassem fatores erosivos em sua dieta, apresentassem alterações significativas em seus perfis de rugosidade. Comprovando nossa hipótese, todas as participantes do grupo A (bruxômanas com e sem erosão) e as participantes do grupo B com fatores erosivos presentes apresentaram aumentos no parâmetro Ra, da ordem de $25,16 \%, 200 \%$ e $74,9 \%$, respectivamente. O grupo B sem erosão foi o único em que a diferença observada não pode ser considerada significativa. Em relação à análise do Ra pode-se observar que os fatores erosivos têm um potencial de degradação maior do que o do bruxismo, e esse potencial se mostra alarmante quando os dois fatores estão associados. Ressalta-se a expressividade do fator erosão, ratificando a importância da avaliação clínica minuciosa, evitando que lesões de desgaste dentário sejam atribuídas apenas ao hábito parafuncional do bruxismo, e/ou mesmo confundidas com este.

Quando comparados os valores de $\mathrm{Ra}$ apresentados pelas participantes do estudo com a diferença de $\mathrm{pH}$ ( $\mathrm{pH}$ final menos o inicial), pode-se mais uma vez perceber que as participantes com fatores sugestivos de erosão apresentaram a maior diferença de valores de pH. De acordo com Ganss (2006) o processo erosivo acarreta não apenas a perda de tecido duro do dente propriamente dito, como torna as superfícies dentárias mais susceptíveis ao desgaste mecânico, o que pode explicar os resultados encontrados neste estudo, onde as participantes que possuíam fatores erosivos presentes (independente se realizassem ou não o bruxismo), apresentaram maiores aumentos no parâmetro $\mathrm{Ra}$.

É válido ressaltar que observando-se individualmente cada participante, podemos perceber que, apesar do cuidado de se realizarem quatro medições durante o período aprovado para a pesquisa, apenas foi possível perceber com clareza a distinção entre o instante inicial $\left(\mathrm{t}_{0}\right)$ e o final $\left(\mathrm{t}_{3}\right)$. O período de tempo avaliado entre as mensurações possivelmente foi curto para que fosse possível detectar mudanças na superfície através do método de perfilometria bidimensional, além de que a expressividade do fenômeno desgaste dentário pode ser distinto para cada indivíduo, havendo momentos de aceleração do processo, e momentos onde o mesmo torna-se mais lento (BARTLETT, 2005). Outra explicação para o achado é que a superfície do esmalte apresenta uma rugosidade natural devido à presença de sulcos, linha de Retzius e pequenos defeitos, que sofrem deposição mineral no ambiente oral (SALAMI; LUZ, 2003). O curto prazo de avaliação das amostras possivelmente captou essas mudanças que ocorrem rotineiramente no ambiente oral, não sendo possível por isso observar uma tendência claramente evolutiva, já que provavelmente o processo de desmineralização não foi consideravelmente maior que o de remineralização a ponto de ser observado a partir da topografia dentária. Apesar das diferenças individuais, o desgaste dentário para que seja percebido na ordem de topografia necessita de um maior período de investigação dentre as mensurações.

Outra forma de descrever o perfil é traçando suas características superficiais em uma curva onde podem ser comparados quantitativa e qualitativamente com outros perfis superficiais, sendo utilizados para isso os parâmetros da família "Rk" (PETROPOULOS, 2007; BASTOS, 2008). A partir de então, como mostrado na Figura 1, é praticamente imperceptível a diferença entre 
as curvas médias de material nos instantes inicial e final para o grupo controle sem erosão. Essa diferença passa a ser percebida para o grupo A sem erosão, e tornase mais evidente no caso do grupo A com erosão. A diferença mais acentuada foi encontrada no grupo onde havia predominância de fatores erosivos. De acordo com Whitehead et al. (1997), casos em que fatores químicos são dominantes, uma combinação de degradação de picos elevados, enfraquecidas pelo ácido, juntamente com um aumento da profundidade de vales, devido à tendência natural dos fluidos para depositar nestas áreas é esperado (WHITEHEAD et al., 1997). Com isso podemos entender porque no grupo onde fatores erosivos estavam presentes a diferença entre as curvas médias de material nos instantes inicial e final foi maior do que no grupo onde além dos fatores erosivos, estavam presentes também o hábito parafuncional do bruxismo.

É possível notar a expressividade do fator erosão dentária não apenas isoladamente, mas também associada ao tão comum hábito parafuncional do bruxismo. Na literatura existe um esforço em caracterizar física e geometricamente as lesões causadas por atrição, abrasão ou por erosão (HOLBROOK; ÁRNADÓTTIR; KAY, 2003; BARTLETT, 2005). No entanto, a falta de uniformidade na terminologia relacionada ao desgaste dentário, erros de tradução (BARDSLEY, 2008) e também a interação de múltiplos fenômenos em sua precipitação tais quais abrasão, atrição e erosão (HOLBROOK; ÁRNADÓTTIR; KAY, 2003; GANSS, 2006), tornam difícil para o profissional a tarefa de determinar a causa principal do desgaste e/ou mesmo avaliar como os fatores em questão têm agido (GANSS, 2006). O que se constatou na prática deste estudo foi uma dificuldade muito grande de diferenciá-las clinicamente a olho nu.

Por isso, indivíduos bruxômanos devem ser orientados e questionados sobre hábitos alimentares e doenças do refluxo gastro-esofágico, assim como profissionais que lidam com o assunto devem estar preparados para compreender e lidar com a fisiopatologia do desgaste dentário, para que possam instituir a terapêutica adequada para cada caso, atingindo com isso a origem do problema, e não apenas tratar seus sintomas. Adicionalmente, na prática clínica, todos os pacientes devem ser considerados como possuindo o risco de desenvolver o desgaste dentário, sendo que uma avaliação criteriosa da superfície dentária deve fazer parte da rotina do profissional (BARTLETT, 2005).

\section{CONCLUSÃO}

Com o presente estudo pode-se verificar, através da análise do perfil de rugosidade de dentes, que o padrão de desgaste quando presentes fatores erosivos é acentuado quando comparado com o desgaste decorrente apenas da atrição.

\section{REFERÊNCIAS}

AHLBERG, J.; RANTALA, M.; SAVOLAINEN, A.; SUVINEN, T.; NISSINEN, M.; SARNA, S.; LINDHOLM, H.; KÖNÖNEN, M. Reported bruxism and stress experience. Community Dent Oral Epidemiol., v. 30, n. 6, p. 405-408. 2002.

BARBOSA, T. S.; MIYAKODA, L.S.; POCZTARUK, R.L.; ROCHA,C.P.; GAVIÃO, M.B. Temporomandibulardisorders and bruxism in childhood and adolescence: Review of the literature. Int J Pediatric Otorhinolaryngol., v. 72, n. 3, p. 299-314. 2008.

BARDSLEY, P. F. The evolution of tooth wear indices. Clin Oral Invest. v. 12, n. 1, p.15-19, 2008.

BARDSLEY, P. F.; TAYLOR, S.; MILOSEVIC, A. Epidemiological studies of tooth wear and dental erosion in 14-year-old children in North West England. Part 1: The relationship with water fluoridation and social deprivation. Br Dent J., v. 197, n. 7, p. 413-416, 2004.

BARTLETT, D. W. The role of erosion in tooth wear: aetiology, prevention and management. I Dent J., v. 55, n. S4, p. 277-284, 2005.

BARTLETT, D. W.; SHAH, P. A Critical Review of Noncarious Cervical (Wear) Lesions and the Role of Abfraction, Erosion, and Abrasion. J Dent Res. v. 85, n. 4, p. 306-312, 2006.

BASTOS, F. S. Análise Numérica do Contato Oclusal, 
Atrito e Desgaste Dentário Humano. 2008. 162f. Tese (Doutorado em Engenharia Mecânica). Escola de Engenharia, Universidade Federal de Minas Gerais, Belo Horizonte.

BHUSHAN, J.; JOSHI, R.; SIDHU, K.; SINGH, A. Tooth Wear - An overview with special emphasis on dental erosion. Indian J Dent Scienc., v. 3, n. 5, p. 89-95, Dec. 2011.

CORRÊA, M. C. C. S. F. Efeitos do refluxo gastroesofágico na cavidade oral. 2007. 117f. Dissertação (Mestrado) Universidade Estadual Paulista, Faculdade de Medicina de Botucatu, 2007.

DALY, R.W.R.; TEE LEE, M.; MUHAMMAD ALI, N. H.; SAMYNATHAN, S.; JIE, Y. P.; ISMAIL, N.H.; HUI YING, Y. B.; SENG, Y. W.; YAHYA, N. A. The study of tooth wear patterns and their associated aetiologies in adults in Kelantan, Malaysia. Archives of Orofacial Sciences. v. 5, n. 2, p. 47-52, 2010.

FIELD, J.; WATERHOUSE, P.; GERMAN, M. Quantifying and qualifying surface changes on dental hard tissues in vitro. J Dentistry. v. 38, n. 3, p. 182-190, 2010.

GANSS, C. Definition of Erosion and Links to Tooth Wear. Monogr Oral Sci. v. 20, p. 9-16, 2006.

GHAZAL, M.; YANG, B.; LUDWIG, K.; KERN, M. Two-body wear of resin and ceramic denture teeth in comparison to human enamel. Dental Materials. v. 24, n. 24, p. 502507, 2008.

GRECHI, T.H.; TRAWITZKI, L.V.; FELÍCIO, C.M.; VALERA, F.C.; ALNSELMO-LIMA, W.T. Bruxism in children with nasal obstruction. Int J Pediatr Otorhinolaryngol., v. 72, n. 3, p. 391-396, 2008.

HEINTZE, S. D.; ZAPPINI, G.; ROUSSON, V. Wear of dental restorative materials in five wear simulators - Results of a round robin test. Dental Materials. n. 21, p. 304-317, 2005.

HOLBROOK, W. P.; ÁRNADÓTTIR, I. B.; KAY, E. J.
Prevention. Part 3: Prevention of tooth wear. Br Dent J., v. 195 , n. 2, p. 75-81, july, 2003.

JOHANSSON, A. K.; OMAR, R.; CARLSSON, G. E.; JOHANSSON, A. Dental erosion and its growing importance in clinical practice: from past to present. Int J Dent., v. 2012, p. 1-17, 2012.

KAIDONIS, J. A.; GRATIAEN, J.; BHATIA, N.; RICHARDS, L.C.; TOWNSEND, G.C. Tooth wear prevention: A quantitative and qualitative in vitro study. Aust Dent J., v. 48 , n. 1, p. 15-19, 2003.

KHAN, F.; YOUNG, W. G.; DALEY, T. J. Dental erosion and bruxism. A tooth wear analysis from South East Queensland. . Aust Dent J., v. 43, n. 2, p. 117-127, 1998.

LAS CASAS, E. B.; SOUZA BASTOS, F. de; BATISTA MEIRELES, A.; Cornacchia, T.P.M.; DURÃES DE GODOY, G.C.; LOPES BUONO, V.T. Enamel canine surface wear evolution in Bruxism. J Biomechanics. v. 39, n. 1, p. S567, 2006.

LOBEZOO, F.; NAEIJE, M. Bruxism is mainly regulated centrally, not peripherally. J Oral Rehabil., v. 28, n. 12, p. 1085-1091, 2001.

LURIE, O.; ZADIK, Y.; EINY, S.; TARRASCH, R.; RAVIV, G.; GOLDSTEIN, L. Bruxism in Military Pilots and NonPilots: Tooth Wear and Psychological Stress. Aviat Space Environ Med., v. 78, n. 2, p. 137-139, Fev. 2007.

MEIRELES, A. B. Métodos para acompanhamento do desgaste dentário no bruxismo. 2009. 85f. Dissertação (Mestrado em Engenharia Mecânica) - Universidade Federal de Minas Gerais, Escola de Engenharia, Belo Horizonte, 2009.

OGLE, R. E.; DAVIS, E. L. Clinical wear study of three commercially available artificial tooth materials: Thirtysix month results. J Prosthet Dent. v. 79, n. 2, p. 145151, fev. 1998.

ORTEGA,A. O. L. Influência de fármacos anticonvulsivantes no Bruxismo de crianças com paralisia cerebral. 2009. 
89f. Tese (Doutorado em Ciências Odontológicas) Universidade de São Paulo, São Paulo, 2009.

PERGAMALIAN, A.; RUDY, T.E.; ZAKI, H.S.; GRECO, C.M. The association between wear facets, bruxism, and severity of facial pain in patients with temporomandibular disorders. J Prosthet Dent., v. 90, n. 2, p. 194-200, Ago. 2003.

PETROPOULOS, G. P. Multi-parameter analysis and modelling of engineering surface texture. J Achiev Mat Manuf Engineering. v. 24, n. 1, p. 91-100, Sep. 2007.

RANJITKAR, S. Biology of tooth wear: preventive strategies. 2009. 105f. Tese (Doutorado em Filosofia) School of Dentistry, Adelaide, 2009.

RIOS, D.; HONÓRIO, H. M.; ARAÚJO, P. A.; MACHADO, M. A. A. M. Wear and superficial roughness of glass ionomer cements used as sealants, after simulated toothbrushing. Pesqui Odontol Bras. v. 16, n. 4, p. 343-348, 2002.

RODRIGUEZ, J. M.; AUSTIN, R. S.; BARTLETT, D. W. A method to evaluate profilometric tooth wear measurements. Dental Materials. v. 28, n. 3, p. 245-251, 2012.

SALAMI, D.; LUZ, M. A. A. C. Effect of prophylactic treatments on the superficial roughness of dental tissues and of two esthetic restorative materials. Pesqui Odontol Bras. v. 17, n. 1, p. 63-68, 2003.

SALVI, R.M.; SILVA, K.S. de; MUTARELLI, P.S.; MACIEL, B.O. Bruxismo associado ao uso de medicamentos. Rev Dor. v. 10, n. 2, p. 169-173, 2009.

SMITH, B. G. N.; BARTLETT, D. W.; ROBB, N. D. The prevalence, etiology and management of tooth wear in the United Kingdom. J Prosthet Dent., v. 78, n. 4, p. 367-372, Oct. 1997.

TAVARES, M. J. G. M.; PRADO JUNIOR, R.R.; MENDES, R.F.; MOITA NETO, J.M. Caracterização do desgaste dental em pacientes acima de 35 anos de idade. RGO, Porto Alegre. v. 55, n. 1, p. 47-53, jan./mar. 2007.
WHITEHEAD, S. A.; LO, L. Y.; WATTS, D. C.; WILSON, N. H. F. Changes of surface texture of enamel in vivo. J Oral Rehab. v. 24, n. 6, p. 449-453, 1997.

WHITEHEAD, S. A.; SHEARER, A. C.; WATTS, D. C.; WILSON, N. H. F. Comparison of two stylus methods for measuring surface texture. Dental Materials. v. 15, n. 2, p. 79-86, 1999.

YOUNG, W. G.; KHAN, F. Sites of dental erosion are salivadependent. J Oral Rehabil., v. 29, n. 1, p. 35-43, 2002.

Recebido em: 27 de maio de 2015 Aceito em: 27 de agosto de 2015 